\title{
Properties of Carathéodory measure hyperbolic universal covers of compact Kähler manifolds
}

\author{
Ngai-fung $N g$
}

\begin{abstract}
This article explores some properties of universal covers of compact Kähler manifolds, under the assumption of Carathéodory measure hyperbolicity. In particular, by comparing invariant volume forms, an inequality is established between the volume of canonical bundle of a compact Kähler manifolds and the Carathéodory measure of its universal cover (similar result as in [Kikuta 10]). Using similar method, an inequality is established between the restricted volume of canonical bundle of a compact Kähler manifolds and the restricted Carathéodory measure of its covering, solving a conjecture in [Kikuta 13].
\end{abstract}

\section{Introduction}

It is interesting to study non-compact complex manifolds with the assumption that there exists bounded holomorphic functions on it, since they include the examples of bounded domains and in particular, Hermitian symmetric spaces of non-compact type. In this article, we shall study properties of universal covers of compact Kähler manifolds, under the assumption of Carathéodory measure hyperbolicity. In this case, there are abundant supplies of holomorphic functions, leading to interesting properties. In [Kikuta 10], it was established that the volume of the canonical line bundle of a compact Kähler manifold is bounded from below by a constant multiple of its Carathéodory measure. It is natural to ask the same question regarding restricted volumes and measures. It was stated as a conjecture in [Kikuta 13] that the restricted volume of the canonical line bundle of a compact Kähler 
manifold is also bounded from below by a constant multiple of its restricted Carathéodory measure. Inspired by the work of [Yeung], which established uniform estimates among invariant metrics, the author obtains uniform estimates among invariant volumes forms and the conjecture follows as an easy consequence.

\section{Invariant Volume Forms}

Let $\mu_{r}^{d}$ be the Poincare volume form for $\mathbb{B}_{r}^{d}$, the complex $d$-ball of radius $r$, i.e.,

$$
\mu_{r}^{d}=\frac{r^{2}}{\left(r^{2}-\|z\|^{2}\right)^{d+1}} \cdot d z^{1} \wedge d \overline{z^{1}} \wedge \cdots \wedge d z^{d} \wedge d \overline{z^{d}}
$$

For the unit ball $\mathbb{B}_{1}^{d}$, we write $\mu_{1}^{d}$ simply as $\mu^{d}$.

Let $M$ be an $n$-dimensional complex manifold. Here we introduce the following invariant volume forms:

The Bergman pseudo-volume form $v_{M}^{B}$ is defined by, for any $p \in M$,

$$
v_{M}^{B}(p):=\sum_{i}\left(\varphi_{i} \wedge \bar{\varphi}_{i}\right)(p)
$$

where $\left\{\varphi_{i}\right\}$ is an orthonormal basis for $L^{2}\left(M, K_{M}\right)$.

From [Hahn], we have

$$
v_{M}^{B}(p)=\varphi_{p} \wedge \overline{\varphi_{p}}
$$

where $\varphi_{p}$ maximizes $(\varphi \wedge \bar{\varphi})(p)$ over the unit ball of $L^{2}\left(M, K_{M}\right)$.

Suppose there exists a Kähler-Einstein metric $g_{M}^{K E}$ on $M$ satisfying

$$
\operatorname{Ric}\left(g_{M}^{K E}\right)=-2(n+1) \cdot \omega_{M}^{K E}
$$

then the Kähler-Einstein volumn form $v_{M}^{K E}$ is defined by

$$
v_{M}^{K E}:=\frac{\left(\omega_{M}^{K E}\right)^{n}}{n !}
$$


The Carathéodory pseudo-volume form $v_{M}^{C}$ is defined by ([Eisenman] page 57), for any $p \in M$,

$$
\begin{aligned}
v_{M}^{C}(p) & :=\sup \left\{\left(f^{*} \mu^{n}\right)(p) ; f: M \rightarrow \mathbb{B}_{1}^{n} \text { holomorphic, } f(p)=0\right\} \\
& =\sup \left\{|J a c(f)(p)|^{2} ; \quad f: M \rightarrow \mathbb{B}_{1}^{n} \text { holomorphic, } f(p)=0\right\}
\end{aligned}
$$

where on any holomorphic coordinate system $\left\{z_{1}, \ldots, z_{n}\right\}$ with $f=\left(f_{1}, \ldots, f_{n}\right)$, we define $J a c(f)$ to be

$$
\left(\begin{array}{cccc}
\frac{\partial f_{1}}{\partial z_{1}} & \frac{\partial f_{1}}{\partial z_{2}} & \ldots & \frac{\partial f_{1}}{\partial z_{n}} \\
\frac{\partial f_{2}}{\partial z_{1}} & \frac{\partial f_{2}}{\partial z_{2}} & \cdots & \frac{\partial f_{2}}{\partial z_{n}} \\
\ldots & \ldots & \cdots & \ldots \\
\frac{\partial f_{n}}{\partial z_{1}} & \frac{\partial f_{n}}{\partial z_{2}} & \cdots & \frac{\partial f_{n}}{\partial z_{n}}
\end{array}\right)
$$

$|J a c(f)|$ to be $\operatorname{det}(J a c(f)) d z^{1} \wedge \cdots \wedge d z^{n}$ and $|J a c(f)|^{2}$ to be

$$
|\operatorname{det}(J a c(f))|^{2} d z^{1} \wedge d \overline{z^{1}} \wedge \cdots \wedge d z^{n} \wedge d \overline{z^{n}}
$$

The Kobayashi pseudo-volume form $v_{M}^{K}$ is defined by ([Eisenman] page 57), for any $p \in M$,

$$
v_{M}^{K}(p):=\inf \left\{|\operatorname{Jac}(f)(0)|^{-2} ; \quad f: \mathbb{B}_{1}^{n} \rightarrow M \text { holomorphic, } f(0)=p\right\}
$$

Next let us consider the case when there is a $d$-dimensional subvariety $Z$ of $M$, in an analogous manner to the above, we may have the following definitions:

The restricted Carathéodory pseudo-volume form $v_{M \mid Z}^{C}$ on the regular locus $Z_{\text {reg }}$ of $Z$ is defined by, for any $p \in Z_{\text {reg }}$,

$$
v_{M \mid Z}^{C}(p):=\sup \left\{\left|J a c\left(\left.f\right|_{Z}\right)(p)\right|^{2} ; \quad f: M \rightarrow \mathbb{B}_{1}^{d} \text { holomorphic, } f(p)=0\right\}
$$

The restricted Kobayashi pseudo-volume form $v_{M \mid Z}^{K}$ on the regular locus $Z_{\text {reg }}$ of $Z$ is defined by, for any $p \in Z_{\text {reg }}$,

$$
v_{M \mid Z}^{K}(p):=\inf \left\{\left|\operatorname{Jac}\left(i_{Z} \circ f\right)(0)\right|^{-2} ; f: \mathbb{B}_{1}^{d} \rightarrow M \text { holomorphic, } f(0)=p\right\}
$$

where $i_{Z}: M \rightarrow Z$ is the restriction map. 


\section{Basic Properties}

The following are the volume decreasing properties of the corresponding volume forms, which are included here for the convenience of the readers.

Proposition 3.1 (Volume decreasing property for Carathéodory measure). Let $N$ be an $n$-dimensional complex manifold. Then, for all $\varphi \in$ $\operatorname{Hol}(M, N)$,

$$
\varphi^{*}\left(v_{N}^{C}\right) \leq v_{M}^{C}
$$

Proof. Any map from $N$ to $\mathbb{B}_{1}^{n}$ induces a map from $M$ to $\mathbb{B}_{1}^{n}$.

Proposition 3.2 (Volume decreasing property for Kobayashi measure). Let $N$ be an $n$-dimensional complex manifold. Then, for all $\varphi \in$ $\operatorname{Hol}(M, N)$,

$$
\varphi^{*}\left(v_{N}^{K}\right) \leq v_{M}^{K}
$$

Proof. Any map from $\mathbb{B}_{1}^{n}$ to $M$ induces a map from $\mathbb{B}_{1}^{n}$ to $N$.

Similarly, we have the following:

Proposition 3.3 (Volume decreasing property for restricted Carathéodory measure). Let $Y$ be an $n$-dimensional complex manifold and $W$ its d-dimensional complex subvariety. Then, for all $\varphi \in \operatorname{Hol}(X, Y)$, such that $\varphi(Z) \subset W$,

$$
\left(\left.\varphi\right|_{Z}\right)^{*}\left(v_{Y \mid W}^{C}\right) \leq v_{X \mid Z}^{C}
$$

Proposition 3.4 (Volume decreasing property for restricted Kobayashi measure). Let $Y$ be an $n$-dimensional complex manifold and $W$ its d-dimensional complex subvariety. Then, for all $\varphi \in \operatorname{Hol}(X, Y)$, such that $\varphi(Z) \subset W$,

$$
\left(\left.\varphi\right|_{Z}\right)^{*}\left(v_{Y \mid W}^{K}\right) \leq v_{X \mid Z}^{K}
$$

We also have continuity property for the Carathéodory volume form.

Theorem 3.5. $v_{M}^{C}$ is locally Lipschitz.

Proof. Let $o \in M$, choose a local coordinate system $U$ at $o$. Let $\mathbb{B}_{r}^{n}$ be the coordinate ball centered at $o$. Let $p, q \in \mathbb{B}_{r / 2}^{n}$ and suppose

$$
v_{M}^{C}(p) \geq v_{M}^{C}(q)
$$


then

$$
\begin{aligned}
v_{M}^{C}(p)-v_{M}^{C}(q) & =\sup |\operatorname{Jac}(f)(p)|^{2}-\sup \frac{|\operatorname{Jac}(f)(q)|^{2}}{\left(1-\|f(q)\|^{2}\right)^{n+1}} \\
& \leq \sup \left\{|\operatorname{Jac}(f)(p)|^{2}-|\operatorname{Jac}(f)(q)|^{2}\right\}
\end{aligned}
$$

where the supremum is taken over $\left\{f: M \rightarrow \mathbb{B}_{1}^{n}\right.$ holomorphic, $\left.f(p)=0\right\}$.

And for all $f: M \rightarrow \mathbb{B}_{1}^{n}$ holomorphic, we have

$$
\begin{aligned}
|\operatorname{Jac}(f)(p)|^{2}-|\operatorname{Jac}(f)(q)|^{2} & \leq(|\operatorname{Jac}(f)(p)-\operatorname{Jac}(f)(q)|)(|\operatorname{Jac}(f)(p)|+|\operatorname{Jac}(f)(q)|) \\
& \leq\left(\frac{n^{1 / 2} 2^{n+2} n !}{r^{n+1}} \cdot\|p-q\|\right)\left(\frac{2^{n+1} n !}{r^{n}}\right) \\
& =\frac{n^{1 / 2} 2^{2 n+3}(n !)^{2}}{r^{2 n+1}} \cdot\|p-q\|
\end{aligned}
$$

by Cauchy inequality applied to $|\operatorname{Jac}(f)|$ and $f$.

Now let $X$ be an $n$-dimensional compact Kähler manifold, $\tilde{X}$ be its universal cover. The next result is a key property of Carathéodory measure hyperbolic universal cover.

Proposition 3.6 ([Kikuta 10]). Suppose $\tilde{X}$ is Carathéodory measure hyperbolic (i.e. $v_{\tilde{X}}^{C}>0$ at every point), then we have $c_{1}\left(K_{X}\right)>0$.

Proof. For any $f: \tilde{X} \rightarrow \mathbb{B}_{1}^{n}$ holomorphic, in a holomorphic coordinate system $\left\{z_{1}, \ldots, z_{n}\right\}, f=\left(f_{1}, \ldots, f_{n}\right)$ and let $\zeta \in \mathbb{C}^{n}$, we have

$$
\begin{aligned}
& \left(\sqrt{-1} \partial \bar{\partial} \log \left(f^{*} \mu^{n}\right)\right)(\zeta, \bar{\zeta}) \\
= & (n+1) \sum_{i, j, k, l}\left(\frac{\left(1-\|f\|^{2}\right) \delta_{i j}+f_{j} \overline{f_{i}}}{\left(1-\|f\|^{2}\right)^{2}}\right) \frac{\partial f_{i}}{\partial z_{k}} \frac{\partial f_{j}}{\partial z_{l}} \zeta^{k} \overline{\zeta^{l}} \\
= & (n+1)\left(\sum_{i, k, l} \frac{1}{1-\|f\|^{2}} \frac{\partial f_{i}}{\partial z_{k}} \frac{\partial f_{i}}{\partial z_{l}} \zeta^{k} \overline{\zeta^{l}}+\frac{1}{\left(1-\|f\|^{2}\right)^{2}}\left|\sum_{i, k} \frac{\partial f_{i}}{\partial z_{k}} \overline{f_{i}} \zeta^{k}\right|^{2}\right) \\
\geq & (n+1) \cdot \frac{1}{1-\|f\|^{2}} \sum_{i, k, l} \frac{\partial f_{i}}{\partial z_{k}} \frac{\partial f_{i}}{\partial z_{l}} \zeta^{k} \overline{\zeta^{l}} \\
= & (n+1) \cdot \frac{1}{1-\|f\|^{2}} \cdot \zeta^{*}(J a c(f))^{*} \cdot J a c(f) \zeta
\end{aligned}
$$


By the Arzela-Ascoli theorem, for any $p \in \tilde{X}$, there is a map $f_{p}: \tilde{X} \rightarrow \mathbb{B}_{1}^{n}$ such that $v_{\tilde{X}}^{C}(p)=\left(f_{p}^{*} \mu^{n}\right)(p)=\left|J a c\left(f_{p}\right)\right|^{2}(p)$. From the above computation we see that locally $\log \left(f_{p}^{*} \mu^{n}\right)$ is strictly plurisubharmonic if and only if $\left|\operatorname{Jac}\left(f_{p}\right)\right| \neq 0$.

Let $p$ be in the local coordinate system and $C$ denote the Lipschitz constant as in the previous Theorem, using the estimates we obtained there, for $q \in B\left(p, \frac{v_{\tilde{X}}^{C}(p)}{10 C}\right):=U$,

$$
\left|\operatorname{Jac}\left(f_{p}\right)\right|^{2}(q)>\left|\operatorname{Jac}\left(f_{p}\right)\right|^{2}(p)-\frac{v_{\tilde{X}}^{C}(p)}{10}=\frac{9}{10} \cdot v_{\tilde{X}}^{C}(p)
$$

That is, on $U,\left|\operatorname{Jac}\left(f_{p}\right)\right|^{2}>\frac{9}{10} \cdot v_{\tilde{X}}^{C}(p)$.

For any $q, s$ in $B\left(p, \frac{v_{\tilde{X}}^{C}(p)}{10 C}\right)$,

$$
\begin{aligned}
& \left.|| \operatorname{Jac}\left(f_{p}\right)\right|^{2}(s)-\left|J a c\left(f_{q}\right)\right|^{2}(s) \mid \\
\leq & \left.|| J a c\left(f_{p}\right)\right|^{2}(s)-\left|J a c\left(f_{p}\right)\right|^{2}(p)|+|\left|J a c\left(f_{p}\right)\right|^{2}(p)-\left|J a c\left(f_{q}\right)\right|^{2}(q)|+|\left|J a c\left(f_{q}\right)\right|^{2}(q)-\left|J a c\left(f_{q}\right)\right|^{2}(s) \mid \\
\leq & \frac{v_{\tilde{X}}^{C}(p)}{10}+\frac{v_{\tilde{X}}^{C}(p)}{10}+\frac{v_{\tilde{X}}^{C}(p)}{5} \\
= & \frac{2}{5} \cdot v_{\tilde{X}}^{C}(p)
\end{aligned}
$$

That is, for all $q \in U,\left|\operatorname{Jac}\left(f_{q}\right)\right|^{2}>\frac{1}{2} \cdot v_{\tilde{X}}^{C}(p)>0$ on $U$.

Notice that $\left.\log \left(v_{\tilde{X}}^{C}\right)\right|_{U}=\sup _{p \in U} \log \left(f_{p}^{*} \mu^{n}\right)$ is continuous and therefore $\log \left(v_{\tilde{X}}^{C}\right)$ is strictly plurisubharmonic.

Interpret $v_{\tilde{X}}^{C}$ as a volume form over $X$ with $\log \left(v_{\tilde{X}}^{C}\right)$ being strictly plurisubharmonic, that defines a strictly positive $(1,1)$ current on $K_{X}$ which can be approximated by a smooth volume form whose curvature form is positive ([Greene-Wu], [Demailly 92]). Hence, $c_{1}\left(K_{X}\right)>0$.

In the case that the covering is a bounded domain in $\mathbb{C}^{n}$, we have the following extra geometric property.

Proposition 3.7 (Uniform Squeezing Property for $\tilde{X}$ ). Suppose $\tilde{X}$ is a bounded domain in $\mathbb{C}^{n}$ which covers a compact complex manifold $X$, then there exists $a, b$ satisfying $0<a<b<\infty$ such that for any $x \in \tilde{X}$, there exists an embedding $\varphi_{x}: \tilde{X} \rightarrow \mathbb{C}^{n}$ with $\varphi_{x}(x)=0$ and $\mathbb{B}_{a}^{n} \subset \varphi_{x}(\tilde{X}) \subset \mathbb{B}_{b}^{n}$. 
Proof. Let $A$ be any fundamental domain of $X$ in $\tilde{X}$. For any $x \in A$, take $r_{x}=\inf _{y \in \partial \tilde{X}}|x-y|>0, R_{x}=\sup _{y \in \partial \tilde{X}}|x-y|<\infty$ so that we have $\mathbb{B}_{r_{x}}^{n}(x) \subset \tilde{X} \subset \mathbb{B}_{R_{x}}^{n}(x)$.

Now since $A$ is relatively compact in $\tilde{X}$, and $r_{x}, R_{x}$ are Lipschitz continuous in $x$, we have $\inf _{A} r_{x}:=a>0$ and $\sup _{A} R_{x}:=b<\infty$.

For any $x \in \tilde{X}-A$, there is an automorphism of $\tilde{X}$ which brings $x$ to a point in $A$. And hence for all points in $\tilde{X}$, we have an embedding $\varphi_{x}$ such that $\varphi_{x}(x)=0$ and $\mathbb{B}_{a}^{n} \subset \varphi_{x}(\tilde{X}) \subset \mathbb{B}_{b}^{n}$.

Finally we include the statement of two Schwarz lemmas and a result on $L^{2}$-estimate for the $\bar{\partial}$-equation that will be used.

Proposition 3.8 (Schwarz lemma of [Mok-Yau]). Let $M$ be a complete Hermitian manifold with scalar curvature bounded from below by $-K_{1}$ and let $N$ be a complex manifold of the same dimension with a volume form $V_{N}$ (i.e., positive $(n, n)$ form, $n=\operatorname{dim} N$ ) such that the Ricci form is negative definite and $\left(\frac{\sqrt{-1}}{2} \partial \bar{\partial} \log V_{N}\right)^{n} \geq K_{2} V_{N}$. Suppose $f: M \rightarrow N$ is a holomorphic map and the Jacobian is nonvanishing at one point. Then $K_{1}>0$ and

$$
\sup \frac{f^{*} V_{N}}{V_{M}} \leq \frac{K_{1}^{n}}{n^{n} K_{2}}
$$

Proposition 3.9 (Schwarz lemma of [Royden]). Let $(M, g)$ be a complete Kähler manifold with Ricci curvature bounded from below by $k \leq 0$, and $(N, h)$ a Kähler manifold with holomorphic sectional curvature bounded from above by $K<0$. Then for any holomorphic map $f: M \rightarrow N$ we have

$$
\sum_{\alpha, \beta, i, j} g^{\alpha \bar{\beta}} f_{\alpha}^{i} \overline{f_{\beta}^{j}} h_{i \bar{j}} \leq \frac{2 \nu}{\nu+1} \frac{k}{K}
$$

where $\nu$ is the maximal rank of $d f$.

Proposition 3.10 ([Hörmander], Theorem 4.1 of [Demailly 82]). Let $M$ be a complete Kähler manifold and let $\varphi$ be a smooth strictly plurisubharmonic function on $M$. Then for any $v \in L_{n, 1}^{2}(M, \partial \bar{\partial} \varphi, \varphi)$ with $\bar{\partial} v=0$, there is an $(n, 0)$-form $u$ on $M$ such that $\bar{\partial} u=v$ and

$$
\left|\int_{M} u \wedge \bar{u} e^{-\varphi}\right| \leq \int_{M}|v|_{\partial \bar{\partial} \varphi}^{2} e^{-\varphi}
$$




\section{Computations on Complex N-ball}

Let $\mathbb{B}_{r}^{n}=\left\{z \in \mathbb{C}^{n}:\|z\|<r\right\}$. Define

$$
\varphi=-\log \left(r^{2}-\|z\|^{2}\right)
$$

and set

$$
\omega=\frac{\sqrt{-1}}{2} \partial \bar{\partial} \varphi=\frac{\sqrt{-1}}{2} \sum_{i, j} g_{i \bar{j}} d z^{i} \wedge d \overline{z^{j}}
$$

Now, Taylor series expansion gives

$-\log \left(r^{2}-\|z\|^{2}\right)=-\log r^{2}+\frac{\|z\|^{2}}{r^{2}}+\frac{\|z\|^{4}}{r^{4}} \cdot \frac{1}{2}+\frac{\|z\|^{6}}{r^{6}} \cdot \frac{1}{3}+\cdots+\frac{\|z\|^{2 n}}{r^{2 n}} \cdot \frac{1}{n}+\cdots$

which shows that

$$
g_{i \bar{j}}(0)=\frac{\delta_{i j}}{r^{2}}
$$

and

$$
d g(0)=0
$$

The curvature tensor is

$$
\begin{aligned}
R_{i \bar{j} k \bar{l}}(0) & =\frac{-\partial^{4} \varphi}{\partial z_{i} \partial \bar{z}_{j} \partial z_{k} \partial \bar{z}_{l}}(0) \\
& =\frac{-1}{2 r^{4}}\left(\frac{\partial^{4}|z|^{4}}{\partial z_{i} \partial \bar{z}_{j} \partial z_{k} \partial \bar{z}_{l}}\right)(0) \\
& =\frac{-1}{2 r^{4}} \cdot \begin{cases}4 & i=j=k=l \\
2 & \{i, k(\neq i)\}=\{j, l\} \\
0 & \text { otherwise }\end{cases}
\end{aligned}
$$

The holomorphic sectional curvature is

$$
\frac{4 \cdot R\left(\partial_{i}, \partial_{\bar{i}}, \partial_{i}, \partial_{\bar{i}}\right)}{\left\|\partial_{i}+\partial_{\bar{i}}\right\|^{4}}(0)=\frac{-8 / r^{4}}{4 / r^{4}}=-2
$$


The Ricci curvature tensor is

$$
\begin{aligned}
\operatorname{Ric}_{k \bar{l}}(0) & =g^{i \bar{j}} R_{i \bar{j} k \bar{l}}(0) \\
& =\sum_{i} r^{2} \cdot R_{\bar{i} \bar{k} k \bar{l}}(0) \\
& = \begin{cases}0 & k \neq l \\
r^{2} \cdot\left[\frac{-4}{2 r^{4}}+\frac{-2}{2 r^{4}} \cdot(n-1)\right] & k=l\end{cases} \\
& = \begin{cases}0 & k \neq l \\
\frac{-(n+1)}{r^{2}} & k=l\end{cases} \\
& = \begin{cases}0 & k \neq l \\
-(n+1) \cdot g_{k \bar{l}}(0) & k=l\end{cases}
\end{aligned}
$$

The scalar curvature is

$$
S(0)=\sum_{k, l} g^{k \bar{l}}(0) \operatorname{Ric}_{k \bar{l}}(0)=\sum_{k} r^{2} \cdot \frac{-(n+1)}{r^{2}}=-n(n+1)
$$

\section{Uniform Estimates among Invariant Vol- umes}

In this section, we are going to establish estimates among various invariant volume forms.

Theorem 5.1. Let $X$ be an n-dimensional compact Kähler manifold, $\tilde{X}$ be its universal cover. Then we have, at any $p \in \tilde{X}$,

(a) [Kobayashi 98]

$$
v_{\tilde{X}}^{C} \leq v_{\tilde{X}}^{K}
$$

(b)

$$
\begin{gathered}
v_{\tilde{X}}^{C} \leq \frac{1}{n !} \cdot v_{\tilde{X}}^{K E} \\
v_{\tilde{X}}^{K E} \leq \frac{1}{n !} \cdot v_{\tilde{X}}^{K} \\
a_{1} \cdot v_{\tilde{X}}^{C} \leq v_{\tilde{X}}^{B} \leq a_{2} \cdot v_{\tilde{X}}^{C}
\end{gathered}
$$

if we assume $\tilde{X}$ is Carathéodory measure hyperbolic, where $a_{1}=a_{1}(X)>$ 0 and $a_{2}=a_{2}(X)<\infty$. 
(c)

$$
v_{\tilde{X}}^{K} \leq \frac{b^{2 n}}{a^{2 n}} \cdot v_{\tilde{X}}^{C}
$$

if we assume $\tilde{X}$ is a bounded domain in $\mathbb{C}^{n}$, where $a=a(X)>0, b=$ $b(X)<\infty$ are as in Proposition (3.7).

Proof.

(a) $v_{\tilde{X}}^{C} \leq v_{\tilde{X}}^{K}$ :

Fix $\epsilon>0, p \in \tilde{X}$. Let $f: \mathbb{B}_{1}^{n} \rightarrow \tilde{X}$ and $g: \tilde{X} \rightarrow \mathbb{B}_{1}^{n}$ be any holomorphic maps with $f(0)=p$ and $g(p)=0$ respectively and such that they satisfy

$$
0<|\operatorname{Jac}(f)(0)|^{-2}<v_{\tilde{X}}^{K}+\epsilon
$$

and

$$
|J a c(g)(p)|^{2}>v_{\tilde{X}}^{C}-\epsilon
$$

Consider the composition $\mathbb{B}_{1}^{n} \stackrel{f}{\rightarrow} \tilde{X} \stackrel{g}{\rightarrow} \mathbb{B}_{1}^{n}$.

Applying Ahlfors-Schwarz lemma (Corollary 2.4.16 of [Kobayashi 98]) gives

$$
(g \circ f)^{*} \mu^{n} \leq \mu^{n}
$$

Expressed in terms of $f$ and $g$ gives

$$
|J a c(g)(p)|^{2} \leq|J a c(f)(0)|^{-2}
$$

and hence

$$
v_{\tilde{X}}^{C}-\epsilon \leq v_{\tilde{X}}^{K}+\epsilon
$$

We are done since $\epsilon$ is arbitrary.

(b) $v_{\tilde{X}}^{C} \leq \frac{1}{n !} \cdot v_{\tilde{X}}^{K E}$ :

By Proposition (3.6), we know that $c_{1}\left(K_{X}\right)>0$.

By [Aubin], [Yau], there is a unique Kähler-Einstein metric $g_{X}^{K E}$ on $X$ such that

$$
\operatorname{Ric}\left(g_{X}^{K E}\right)=-2(n+1) \cdot \omega_{X}^{K E}
$$


Pull back the metric by $\pi$ so that we have, on $\tilde{X}$, the complete metric $g_{\tilde{X}}^{K E}$ satisfying

$$
\operatorname{Ric}\left(g_{\tilde{X}}^{K E}\right)=-2(n+1) \cdot \omega_{\tilde{X}}^{K E}
$$

Hence the scalar curvature of $\tilde{X}$ w.r.t this metric is $-n(n+1)$.

On $\mathbb{B}_{1}^{n}$, we can construct a Kähler-Einstein metric $g_{\mathbb{B}_{1}^{n}}^{K E}$ such that

$$
\operatorname{Ric}\left(g_{\mathbb{B}_{1}^{n}}^{K E}\right)=-2(n+1) \cdot \omega_{\mathbb{B}_{1}^{n}}^{K E}
$$

Hence

$$
\left(\frac{\sqrt{-1}}{2} \partial \bar{\partial} \log \left(\mu^{n}\right)\right)^{n}=(n+1)^{n} n ! \cdot \mu^{n}
$$

For any map $f: \tilde{X} \rightarrow \mathbb{B}_{1}^{n}$, applying the Schwarz lemma of [Mok-Yau] yields

$$
f^{*} \mu^{n} \leq \frac{(n(n+1))^{n}}{n^{n}(n+1)^{n} n !} \cdot v_{\tilde{X}}^{K E}=\frac{1}{n !} \cdot v_{\tilde{X}}^{K E}
$$

Hence we have

$$
v_{\tilde{X}}^{C} \leq \frac{1}{n !} \cdot v_{\tilde{X}}^{K E}
$$

(b) $v_{\tilde{X}}^{K E} \leq \frac{1}{n !} \cdot v_{\tilde{X}}^{K}$ :

For any $p \in \tilde{X}$, consider any map $f: \mathbb{B}_{1}^{n} \rightarrow \tilde{X}$ with $f(0)=p$. Applying the Schwarz lemma of [Mok-Yau] yields

$$
f^{*} v_{\tilde{X}}^{K E} \leq \frac{(n(n+1))^{n}}{n^{n}(n+1)^{n} n !} \cdot \mu^{n}
$$

Hence we have

$$
v_{\tilde{X}}^{K E} \leq \frac{1}{n !} \cdot v_{\tilde{X}}^{K}
$$

(b) $a_{1} \cdot v_{\tilde{X}}^{C} \leq v_{\tilde{X}}^{B} \leq a_{2} \cdot v_{\tilde{X}}^{C}$ :

Recall that for any $p \in \tilde{X}$, there is an $f_{p}: \tilde{X} \rightarrow \mathbb{B}_{1}^{n}$ such that $f_{p}(p)=0$ and $v_{\tilde{X}}^{C}=\left|\operatorname{Jac}\left(f_{p}\right)\right|^{2}>0$. Let us first construct a bounded smooth strictly plurisubharmonic function $\psi$ on $\tilde{X}$. Pick any $p_{1}$ in $\tilde{X}$, $\left|\operatorname{Jac}\left(f_{p_{1}}\right)\right| \neq 0$ except perhaps on an $(n-1)$-dimensional subvariety $Z_{1}$. Pick a set of $p_{2 i}$ 's in the connected components of $Z_{1}$, then $\left|J a c\left(f_{p_{1}}\right)\right|$ 
and $\left|\operatorname{Jac}\left(f_{p_{2 i}}\right)\right|$ 's all $\neq 0$ except perhaps on a strictly lower dimensional subvariety $Z_{2}$ than $Z_{1}$. Inductively, we will find a countable collection of $\left\{f_{q_{1}}, \ldots, f_{q_{n}}, \ldots\right\}$ such that their Jacobian determinant is not simultaneously zero. Consider the function $\psi:=\log \left(\sum_{i=1}^{\infty} \frac{1}{2^{i}}\left\|f_{q_{i}}\right\|^{2}\right)$. A straight forward computation shows that $\psi$ is plurisubharmonic and by our choice of the $f$ 's, we know that $\psi$ is indeed strictly plurisubharmonic. Clearly $\psi$ is also bounded.

Let $\lambda$ be a $C^{\infty}$ real smooth cut off function that is $\equiv 1$ in a neighborhood of $p$. Choose $k>0$ such that $\varphi:=k \psi+\lambda(n+1) \log |z|^{2}$ is strictly psh on $\tilde{X} \backslash\{p\}$. By Proposition (3.10), we can solve the equation $\bar{\partial} u=\bar{\partial}\left(\lambda\left|J a c\left(f_{p}\right)\right|\right)$ on $\tilde{X} \backslash\{p\}$ with estimate

$$
\left|\int_{\tilde{X} \backslash\{p\}} u \wedge \bar{u} e^{-\varphi}\right| \leq \int_{\tilde{X} \backslash\{p\}}\left|\bar{\partial}\left(\lambda\left|\operatorname{Jac}\left(f_{p}\right)\right|\right)\right|_{\partial \bar{\partial} \varphi}^{2} e^{-\varphi} \leq C
$$

This implies that the $L^{2}$ holomorphic $n$-form $v=\lambda\left|\operatorname{Jac}\left(f_{p}\right)\right|-u$ satisfies

$$
v(p)=\left|\operatorname{Jac}\left(f_{p}\right)\right|(p) \neq 0
$$

because from the choice of $\varphi$ we know that $u(p)=0$. Hence, $v_{\tilde{X}}^{B}(p)>$ 0 . Consider the ratio $v_{\tilde{X}}^{B} / v_{\tilde{X}}^{C}$, being continuous and invariant under automorphisms of $\tilde{X}$, let $A$ be a fundamental domain of $X$ in $\tilde{X}$, we have

$$
\inf _{\tilde{X}} v_{\tilde{X}}^{B} / v_{\tilde{X}}^{C}=\inf _{A} v_{\tilde{X}}^{B} / v_{\tilde{X}}^{C}:=a_{1}(X)>0
$$

and

$$
\sup _{\tilde{X}} v_{\tilde{X}}^{B} / v_{\tilde{X}}^{C}=\sup _{A} v_{\tilde{X}}^{B} / v_{\tilde{X}}^{C}:=a_{2}(X)<\infty
$$

(c) $v_{\tilde{X}}^{K} \leq \frac{b^{2 n}}{a^{2 n}} \cdot v_{\tilde{X}}^{C}$ :

Realize $\tilde{X}$ as $\mathbb{B}_{a}^{n} \subset \tilde{X} \subset \mathbb{B}_{b}^{n}$ with $p=(0, \ldots, 0)$. Consider the composition

$$
\mathbb{B}_{1}^{n} \rightarrow \mathbb{B}_{a}^{n} \hookrightarrow \tilde{X} \hookrightarrow \mathbb{B}_{b}^{n} \rightarrow \mathbb{B}_{1}^{n}
$$

in which the first and last maps are given, respectively, by

$$
\left(w_{1}, \ldots, w_{n}\right) \mapsto\left(a w_{1}, \ldots, a w_{n}\right)
$$


and

$$
\left(z_{1}, \ldots, z_{n}\right) \mapsto\left(z_{1} / b, \ldots, z_{n} / b\right)
$$

Hence,

$$
v_{\tilde{X}}^{C}(p) \geq \frac{1}{b^{2 n}} \cdot d z^{1} \wedge d \overline{z^{1}} \wedge \cdots \wedge d z^{n} \wedge d \overline{z^{n}}
$$

and

$$
v_{\tilde{X}}^{K}(p) \leq \frac{1}{a^{2 n}} \cdot d z^{1} \wedge d \overline{z^{1}} \wedge \cdots \wedge d z^{n} \wedge d \overline{z^{n}}
$$

We know that the Carathéodory pseudo-volume form is invariant under biholomorphic map. Hence, the Carathéodory measure $\mu_{\tilde{X}}^{C}(X)$ of $X$ can thus be defined to be

$$
\mu_{\tilde{X}}^{C}(X):=\int_{A} v_{\tilde{X}}^{C}
$$

where $A$ can be any fundamental domain of $X$ in $\tilde{X}$.

Next, let $L$ be a holomorphic line bundle over $X$, the volume $\operatorname{vol}_{X}(L)$ of $L$ is defined as

$$
\operatorname{vol}_{X}(L):=\limsup _{m \rightarrow \infty} \frac{\operatorname{dim} H^{0}(X, \mathcal{O}(m L))}{m^{n} / n !}
$$

If $L>0$, by the Hirzebruch-Riemann-Roch formula and Kodaira vanishing theorem, we have

$$
\operatorname{vol}_{X}(L)=\int_{X} c_{1}(L)^{n}
$$

Corollary 5.2. Let $X$ be an n-dimensional compact Kähler manifold such that $\tilde{X}$ is Carathéodory measure hyperbolic, then we have

$$
\frac{(n !)^{2}(n+1)^{n}}{(\pi)^{n}} \cdot \mu_{\tilde{X}}^{C}(X) \leq \operatorname{vol}_{X}\left(K_{X}\right)
$$

Proof. From

$$
n ! \cdot v_{\tilde{X}}^{C} \leq v_{\tilde{X}}^{K E}=\frac{1}{n !}\left(\omega_{\tilde{X}}^{K E}\right)^{n}=\frac{1}{n !(2(n+1))^{n}}\left(-\operatorname{Ric}\left(g_{\tilde{X}}^{K E}\right)\right)^{n}
$$

we have

$$
\frac{(n !)^{2}(n+1)^{n}}{(\pi)^{n}} \cdot v_{\tilde{X}}^{C} \leq\left(-\frac{1}{2 \pi} \operatorname{Ric}\left(g_{\tilde{X}}^{K E}\right)\right)^{n}
$$


Let $A$ be a fundamental domain of $X$ in $\tilde{X}$. Integrate the above inequality over $A$, we have

$$
\frac{(n !)^{2}(n+1)^{n}}{(\pi)^{n}} \int_{A} v_{\tilde{X}}^{C} \leq \int_{A}\left(-\frac{1}{2 \pi} \operatorname{Ric}\left(g_{\tilde{X}}^{K E}\right)\right)^{n}
$$

Since $c_{1}\left(K_{X}\right)>0$, we get

$$
\frac{(n !)^{2}(n+1)^{n}}{(\pi)^{n}} \cdot \mu_{\tilde{X}}^{C}(X) \leq \operatorname{vol}_{X}\left(K_{X}\right)
$$

Similar estimate is obtained in [Kikuta 10] by using a different method.

\section{Uniform Estimates among Restricted In- variant Volumes}

Similar to the treatment of the previous section, we have the following:

Theorem 6.1. Let $X$ be an $n$-dimensional compact Kähler manifold, $\pi$ : $\tilde{X} \rightarrow X$ be its universal cover, $Z$ be a d-dimensional subvariety of $X, \tilde{Z}_{\text {reg }}$ is the regular part of the subvariety $\tilde{Z}:=\pi^{-1}(Z)$. Then we have, at any $p \in \tilde{Z}_{\text {reg }}$,

(a)

$$
v_{\tilde{X} \mid \tilde{Z}}^{C} \leq v_{\tilde{X} \mid \tilde{Z}}^{K}
$$

(b)

$$
v_{\tilde{X} \mid \tilde{Z}}^{C} \leq \frac{d^{d}(n+1)^{d}}{(d+1)^{d}} \cdot v_{\tilde{X} \mid \tilde{Z}}^{K E}
$$

if we assume $\tilde{X}$ is Carathéodory measure hyperbolic.

(c)

$$
v_{\tilde{X} \mid \tilde{Z}}^{K} \leq \frac{b^{2 d}}{a^{2 d}} \cdot v_{\tilde{X} \mid \tilde{Z}}^{C}
$$

if we assume $\tilde{X}$ is a bounded domain in $\mathbb{C}^{n}$, where $a=a(X), b=b(X)$ are as in Proposition (3.7). 
Proof.

(a) $v_{\tilde{X} \mid \tilde{Z}}^{C} \leq v_{\tilde{X} \mid \tilde{Z}}^{K}:$

Fix $\epsilon>0, p \in \tilde{Z}_{\text {reg. }}$ Let $f: \mathbb{B}_{1}^{d} \rightarrow \tilde{X}$ and $g: \tilde{X} \rightarrow \mathbb{B}_{1}^{d}$ be any holomorphic maps with $f(0)=p$ and $g(p)=0$ respectively and such that they satisfy

$$
0<\left|\operatorname{Jac}\left(i_{\tilde{Z}_{r e g}} \circ f\right)(0)\right|^{-2}<v_{\tilde{X} \mid \tilde{Z}}^{K}+\epsilon
$$

and

$$
\left|\operatorname{Jac}\left(\left.g\right|_{\tilde{Z}_{r e g}}\right)(p)\right|^{2}>v_{\tilde{X} \mid \tilde{Z}}^{C}-\epsilon
$$

Consider the composition $\mathbb{B}_{1}^{d} \stackrel{f}{\rightarrow} \tilde{X} \stackrel{i_{\tilde{Z}_{\text {reg }}}^{\longrightarrow}}{\longrightarrow} \tilde{Z}_{\text {reg }} \stackrel{\left.g\right|_{\tilde{Z}_{r e g}}}{\longrightarrow} \mathbb{B}_{1}^{d}$.

Applying Ahlfors-Schwarz lemma (Corollary 2.4.16 of [Kobayashi 98]) gives

$$
(g \circ f)^{*} \mu^{d} \leq \mu^{d}
$$

Expressed in terms of $i_{\tilde{Z}_{\text {reg }}} \circ f$ and $\left.g\right|_{\tilde{Z}_{\text {reg }}}$ gives

$$
\left|\operatorname{Jac}\left(\left.g\right|_{\tilde{Z}_{r e g}}\right)(p)\right|^{2} \leq\left|\operatorname{Jac}\left(i_{\tilde{Z}_{r e g}} \circ f\right)(0)\right|^{-2}
$$

and hence

$$
v_{\tilde{X} \mid \tilde{Z}}^{C}-\epsilon \leq v_{\tilde{X} \mid \tilde{Z}}^{K}+\epsilon
$$

We are done since $\epsilon$ is arbitrary.

(b) $v_{\tilde{X} \mid \tilde{Z}}^{C} \leq \frac{d^{d}(n+1)^{d}}{(d+1)^{d}} \cdot v_{\tilde{X} \mid \tilde{Z}}^{K E}$ :

By Proposition (3.6), we know that $c_{1}\left(K_{X}\right)>0$.

By [Aubin], [Yau], there is a unique Kähler-Einstein metric $g_{X}^{K E}$ on $X$ such that

$$
\operatorname{Ric}\left(g_{X}^{K E}\right)=-2(n+1) \cdot \omega_{X}^{K E}
$$

Pull back the metric by $\pi$ so that we have, on $\tilde{X}$, the complete metric $g_{\tilde{X}}^{K E}$ satisfying

$$
\operatorname{Ric}\left(g_{\tilde{X}}^{K E}\right)=-2(n+1) \cdot \omega_{\tilde{X}}^{K E}
$$

On $\mathbb{B}_{1}^{d}$, we can construct a Kähler-Einstein metric $g_{\mathbb{B}_{1}^{d}}^{K E}$ such that

$$
\operatorname{Ric}\left(g_{\mathbb{B}_{1}^{d}}^{K E}\right)=-2(d+1) \cdot \omega_{\mathbb{B}_{1}^{d}}^{K E}
$$


For any map $f: \tilde{X} \rightarrow \mathbb{B}_{1}^{d}$, applying the Schwarz lemma of [Royden] yields

$$
f^{*} \omega_{\mathbb{B}_{1}^{d}}^{K E} \leq \frac{2 d}{d+1} \frac{n+1}{2} \cdot \omega_{\tilde{X}}^{K E}
$$

Restricting to $\tilde{Z}_{\text {reg }}$ and taking $d$-th power on both sides yields

$$
f^{*} \mu^{d} \leq \frac{d^{d}(n+1)^{d}}{(d+1)^{d}} \cdot \frac{\left(\omega_{\tilde{X}}^{K E}\right)^{d}}{d !}
$$

and therefore

$$
v_{\tilde{X} \mid \tilde{Z}}^{C} \leq \frac{d^{d}(n+1)^{d}}{(d+1)^{d}} \cdot v_{\tilde{X} \mid \tilde{Z}}^{K E}
$$

(c) $v_{\tilde{X} \mid \tilde{Z}}^{K} \leq \frac{b^{2 d}}{a^{2 d}} \cdot v_{\tilde{X} \mid \tilde{Z}}^{C}$ :

Realize $\tilde{X}$ as $\mathbb{B}_{a}^{n} \subset \tilde{X} \subset \mathbb{B}_{b}^{n}$ with $p=(0, \ldots, 0)$ and locally at $p, \tilde{Z}$ is given by $z_{d+1}=\ldots=z_{n}=0$. Consider the composition

$$
\mathbb{B}_{1}^{d} \rightarrow \mathbb{B}_{a}^{n} \hookrightarrow \tilde{X} \hookrightarrow \mathbb{B}_{b}^{n} \rightarrow \mathbb{B}_{1}^{d}
$$

in which the first and last maps are given, respectively, by

$$
\left(w_{1}, \ldots, w_{d}\right) \mapsto\left(a w_{1}, \ldots, a w_{d}, 0, \ldots, 0\right)
$$

and

$$
\left(z_{1}, \ldots, z_{n}\right) \mapsto\left(z_{1} / b, \ldots, z_{d} / b\right)
$$

Hence,

$$
v_{\tilde{X} \mid \tilde{Z}}^{C}(p) \geq \frac{1}{b^{2 d}} \cdot d z^{1} \wedge d \overline{z^{1}} \wedge \cdots \wedge d z^{n} \wedge d \overline{z^{n}}
$$

and

$$
v_{\tilde{X} \mid \tilde{Z}}^{K}(p) \leq \frac{1}{a^{2 d}} \cdot d z^{1} \wedge d \overline{z^{1}} \wedge \cdots \wedge d z^{n} \wedge d \overline{z^{n}}
$$

We know that the restricted Carathéodory pseudo-volume form is invariant under biholomorphic map preserving $Z$. Hence, the restricted Carathéodory measure $\mu_{\tilde{X} \mid \tilde{Z}}^{C}(Z)$ of $Z$ can thus be defined to be

$$
\mu_{\tilde{X} \mid \tilde{Z}}^{C}(Z):=\int_{A \cap \tilde{Z}_{r e g}} v_{\tilde{X} \mid \tilde{Z}}^{C}
$$


where $A$ can be any fundamental domain of $X$ in the covering $\pi: \tilde{X} \rightarrow X$ and $\tilde{Z}_{\text {reg }}$ is the regular part of the subvariety $\tilde{Z}:=\pi^{-1}(Z)$.

Next, let $L$ be a holomorphic line bundle over $X$, the restricted volume $\operatorname{vol}_{X \mid Z}(L)$ of $L$ along $Z$ is defined as

$$
\begin{gathered}
\operatorname{vol}_{X \mid Z}(L):=\limsup _{m \rightarrow \infty} \frac{\operatorname{dim} H^{0}(X \mid Z, \mathcal{O}(m L))}{m^{d} / d !} \\
:=\limsup _{m \rightarrow \infty} \frac{\operatorname{dim} \operatorname{Im}\left[i_{Z}^{*}: H^{0}(X, m L) \rightarrow H^{0}\left(Z,\left.m L\right|_{Z}\right)\right]}{m^{d} / d !}
\end{gathered}
$$

The following result will be needed to estimate the restricted volume of $K_{X}$.

Proposition 6.2 ([Boucksom],[Hisamoto],[Matsumura]). Let $X$ be an $n$ dimensional projective manifold, $L$ a big line bundle over $X$ (i.e. its Kodaira dimension is $n$ ), and $Z$ an irreducible closed d-dimensional subvariety of $X$. Furthermore, suppose that $Z \not \subset \mathbb{B}_{+}(L)$. Then

$$
\operatorname{vol}_{X \mid Z}(L)=\sup _{T}\left\{\int_{Z_{r e g}}\left(\left.T\right|_{Z_{r e g}}\right)_{a c}^{d}\right\}
$$

where the supremum is taken over all $T$ 's that are semi-positive $(1,1)$-current in $c_{1}(L)$ that have measure zero unbounded loci and whose unbounded loci do not contain $Z$. Here we denote $\left.T\right|_{Z_{\text {reg }}}$ the restriction of $T$ to the regular locus of $Z$ and $\left(\left.T\right|_{Z_{\text {reg }}}\right)_{\text {ac }}$ its absolutely continuous part.

Here we arrive at our promised result, settling a conjecture in [Kikuta 13].

Corollary 6.3. Let $X$ be an n-dimensional compact Kähler manifold such that $\tilde{X}$ is Carathéodory measure hyperbolic, $Z$ be a d-dimensional subvariety of $X$, then we have

$$
\frac{d !(d+1)^{d}}{\pi^{d} d^{d}} \cdot \mu_{\tilde{X} \mid \tilde{Z}}^{C}(Z) \leq \operatorname{vol}_{X \mid Z}\left(K_{X}\right)
$$

Proof. From

$$
\frac{(d+1)^{d}}{d^{d}(n+1)^{d}} \cdot v_{\tilde{X} \mid \tilde{Z}}^{C} \leq v_{\tilde{X} \mid \tilde{Z}}^{K E}=\frac{1}{d !}\left(\left.\left(\omega_{\tilde{X}}^{K E}\right)\right|_{\tilde{Z}_{r e g}}\right)^{d}=\frac{1}{d !(2(n+1))^{d}}\left(-\left.\operatorname{Ric}\left(g_{\tilde{X}}^{K E}\right)\right|_{\tilde{Z}_{r e g}}\right)^{d}
$$


we have

$$
\frac{d !(d+1)^{d}}{\pi^{d} d^{d}} \cdot v_{\tilde{X} \mid \tilde{Z}}^{C} \leq\left(-\left.\frac{1}{2 \pi} \operatorname{Ric}\left(g_{\tilde{X}}^{K E}\right)\right|_{\tilde{Z}_{r e g}}\right)^{d}
$$

Let $A$ be a fundamental domain of $X$ in $\tilde{X}$. Integrate the above inequality over $A \cap \tilde{Z}_{\text {reg }}$ gives

$$
\frac{d !(d+1)^{d}}{\pi^{d} d^{d}} \int_{A \cap \tilde{Z}_{r e g}} v_{\tilde{X} \mid \tilde{Z}}^{C} \leq \int_{A \cap \tilde{Z}_{r e g}}\left(-\left.\frac{1}{2 \pi} \operatorname{Ric}\left(g_{\tilde{X}}^{K E}\right)\right|_{\tilde{Z}}\right)^{d}
$$

Apply Proposition (6.2), we get

$$
\frac{d !(d+1)^{d}}{\pi^{d} d^{d}} \cdot \mu_{\tilde{X} \mid \tilde{Z}}^{C}(Z) \leq \operatorname{vol}_{X \mid Z}\left(K_{X}\right)
$$

\section{Completeness of Bergman Metric}

Suppose that $v_{M}^{B}>0$ at every point, the Bergman pseudometric $g_{M}^{B}$ on $M$ is defined by

$$
\left(g_{M}^{B}\right)_{i \bar{j}}:=\frac{\partial^{2} \log \left(v_{M}^{B}\right)}{\partial z_{i} \partial \overline{z_{j}}}
$$

From [Hahn], we have

$$
g_{M}^{B}(p, v)=\frac{\partial_{v} g_{p, v} \wedge \overline{\partial_{v} g_{p, v}}}{\varphi_{p} \wedge \overline{\varphi_{p}}}
$$

where $\varphi_{p}$ maximizes $(\varphi \wedge \bar{\varphi})(p)$ and $g_{p, v}$ maximizes $\left(\partial_{v} g \wedge \overline{\partial_{v} g}\right)(p)$ with $g(p)=0$ over the unit ball of $L^{2}\left(M, K_{M}\right)$.

Theorem 7.1. Let $X$ be an n-dimensional compact Kähler manifold such that $\tilde{X}$ is Carathéodory measure hyperbolic, then $g_{\tilde{X}}^{B}$ is complete.

Proof. By Theorem (5.1), we know that $v_{\tilde{X}}^{B}>0$ at every point, so $g_{\tilde{X}}^{B}$ is welldefined. By Proposition (3.6), we know that $c_{1}\left(K_{X}\right)>0$, hence there exists a complete Kähler-Einstein metric on $\tilde{X}$. By Proposition 2.3 of [Chen], to show that the Bergman metric is positive definite, it suffices to construct a bounded smooth strictly plurisubharmonic function $\psi$ on $\tilde{X}$. As is discussed 
in the proof of Theorem (5.1), such a function $\psi$ exists.

Now let $\left\{y_{i}\right\}$ be a Cauchy sequence in $\tilde{X}$ w.r.t. $g_{\tilde{X}}^{B}$. As $g_{\tilde{X}}^{B}$ is an invariant metric, the push-forward of $g_{\tilde{X}}^{B}$ by $\pi: \tilde{X} \rightarrow X$ onto $X$ is a well-defined, positive definite metric, denoted by $g_{X}$. Hence, $\left\{\pi\left(y_{i}\right)\right\}$ is Cauchy w.r.t. $g_{X}$ on the compact manifold $X$. Hence, $\left\{\pi\left(y_{i}\right)\right\}$ converges to, say, $z \in X$. Let $\pi^{-1}\{z\}=\left\{x_{i}\right\}_{i \in I}$. By the discreteness of the action of deck transformations on $\left\{x_{i}\right\}_{i \in I}$, there exists $\epsilon$-neigborhoods of the $x_{i}$ 's that are mutually nonintersecting, hence, there exists $x_{i_{0}}$ in $\left\{x_{i}\right\}_{i \in I}$ that is the limit of $\left\{y_{i}\right\}$.

\section{Invariant metrics}

For a tangent vector $v \in T_{p} M$ on a complex manifold $M$, the Kobayashi and Carathéodory pseudometrics are defined by

$$
\begin{aligned}
& \sqrt{g_{M}^{C}(p, v)}=\sup \left\{\|u\| ; \quad f: M \rightarrow \mathbb{B}_{1}^{1} \text { holomorphic, } f(p)=0, f_{*} v=u\right\} \\
& \sqrt{g_{M}^{K}(p, v)}=\inf \left\{\|u\| ; \quad f: \mathbb{B}_{1}^{1} \rightarrow M \text { holomorphic, } f(0)=p, f_{*} u=v\right\}
\end{aligned}
$$

The following theorem includes various estimates established among invariant metrics, they are included for the convenience of the readers. The only part that is new is part(b), which is proved by a simple application of Schwarz lemma of [Royden].

Theorem 8.1. Let $X$ be an $n$-dimensional compact Kähler manifold, $\tilde{X}$ be its universal cover. Then we have, at any $p \in \tilde{X}$ and $v \in T_{p} \tilde{X}$,

(a)

$$
\begin{aligned}
& g_{\tilde{X}}^{C} \leq g_{\tilde{X}}^{K} \\
& g_{\tilde{X}}^{C} \leq g_{\tilde{X}}^{B}
\end{aligned}
$$

$$
g_{\tilde{X}}^{C} \leq(n+1) \cdot g_{\tilde{X}}^{K E}
$$

if we assume $\tilde{X}$ is Carathéodory measure hyperbolic. 
(c)

$$
\begin{gathered}
g_{\tilde{X}}^{K} \leq \frac{b^{2}}{a^{2}} \cdot g_{\tilde{X}}^{C} \\
g_{\tilde{X}}^{B} \leq\left[\frac{2 \pi}{a^{3}}\left(\frac{2 b}{a}\right)^{n}\right]^{2} g_{\tilde{X}}^{K} \\
\frac{a^{2}}{b^{2} n} g_{\tilde{X}}^{K} \leq g_{\tilde{X}}^{K E} \leq \frac{b^{4 n-2} n^{n-1}}{a^{2 n-2}} g_{\tilde{X}}^{K}
\end{gathered}
$$

if we assume $\tilde{X}$ is a bounded domain in $\mathbb{C}^{n}$, where $a=a(X), b=b(X)$ are as in Proposition (3.7).

Proof.

(a) $g_{\tilde{X}}^{C} \leq g_{\tilde{X}}^{K}$ :

It follows directly from the Schwarz lemma.

(a) $g_{\tilde{X}}^{C} \leq g_{\tilde{X}}^{B}$ :

See [Hahn].

(b) $g_{\tilde{X}}^{C} \leq(n+1) \cdot g_{\tilde{X}}^{K E}$ :

For any map $f: \tilde{X} \rightarrow \mathbb{B}_{1}^{1}$, applying the Schwarz lemma of [Royden] yields

$$
f^{*} \mu^{1} \leq \frac{2(1)(-(2 n+2))}{(1+1)(-2)} \cdot g_{\tilde{X}}^{K E}
$$

Hence we have

$$
g_{\tilde{X}}^{C} \leq(n+1) \cdot g_{\tilde{X}}^{K E}
$$

(c) See [Yeung]. 


\section{References}

[Aubin] T. Aubin, Equations du type Monge-Ampere sur les varietes kähleriennes compactes, Bull. Sc. Math. 102, (1978) 63-95.

[Boucksom] S. Boucksom, On the volume of a line bundle, Internat. J. Math. 13 (2002), 1043-1063.

[Chen] B.-Y. Chen, The Bergman metric on complete Kähler manifolds, Math. Ann. 327 (2003), no. 2, 339-349.

[Demailly 82] J. P. Demailly, Estimations $L^{2}$ pour l'opérateur d'un fibré vectoriel holomorphic semi-positiv au dessus d'une variété kählérienne complète, Ann. Sci. Ec. Norm. Sup. 15, 457-511 (1982)

[Demailly 92] J. P. Demailly, Regularization of closed positive currents and Intersection Theory, J. Algebraic Geom. 1 (1992) no. 3, 361-409.

[Eisenman] D. A. Eisenman, Intrinsic measures on complex manifolds and holomorphic mappings, Memoirs of the American Math. Soc. No. 96 (1970).

[Ein-Lazarsfeld-Mustata-Nakamaye-Popa] L. Ein, R. Lazarsfeld, M. Mustata, M. Nakamaye, M. Popa, Restricted Volumes and Base Loci of Linear Series, American Journal of Mathematics 131 (2009), 607-651.

[Greene-Wu] R. E. Greene, H. Wu, $C^{\infty}$ approximations of convex, subharmonic, and plurisubharmonic functions, Annales scientifiques de l'É.N.S. $4^{e}$ série, tome 12 , no. 1 (1979), p.47-84.

[Hahn] K. T. Hahn, Inequality between the Bergman metric and Carathéodory differential metric, Proceedings of Amer. Math. Soc., Volume 68, Number 2 (1978), 193-194.

[Hisamoto] T. Hisamoto, Restricted Bergman kernel asymptotics, Trans. Amer. Math. Soc. 364 (2012), 3585-3607.

[Hörmander] L. Hörmander, $L^{2}$ estimates and existence theorems for the $\bar{\partial}$ operator, Acta Math. 113 (1965), 89-152 
[Kikuta 10] S. Kikuta, Carathéodory Measure Hyperbolicity and Positivity of Canonical Bundles, Proceedings of the American Mathematical Society, Volume 139, Number 4, April 2011, Pages 1411-1420. Electronically Published on September 1, 2010.

[Kikuta 13] S. Kikuta, Restricted Carathéodory Measure and Restricted Volume of the Canonical Bundle, Michigan Math. J. 62 (2013), 259-292.

[Kobayashi 59] S. Kobayashi, Geometry of Bounded Domains, Trans. Amer. Math. Soc. 92 (1959), 267-290.

[Kobayashi 98] S. Kobayashi, Hyperbolic Complex Spaces, Springer Verlag (1998).

[Liu-Sun-Yau] K. Liu, X. Sun, and S.-T. Yau, Canonical metrics on the moduli space of Riemann Surfaces I, J. Differential Geom. Volume 68, Number 3 (2004), 571-637.

[Matsumura] S. Matsumura, Restricted volumes and divisorial Zariski decompositions, Amer. J. Math. (Impact Factor: 1.18). 05/2010; 135(3).

[Mok-Yau] N. Mok, S.-T. Yau, Completeness of the Káhler-Einstein metric on bounded domains and the characterization of domains of holomorphy by curvature conditions, in: The Mathematical Heritage of Henri Poincaré, part 1, Bloomington, IN, 1980, in: Proc. Sympos. Pure Math., vol. 39, Amer. Math. Soc., Providence, RI, 1983, pp. 41-59.

[Richberg] R. Richberg, Stetie streng pseudokonvexe Funktionen, Math. Ann., 175 (1968), 257-286

[Royden] H. L. Royden, The Ahlfors-Schwarz lemma in several complex variables, Comment. Math. Helvetici 55 (1980), 547-558

[Yau] S.-T. Yau, On the Ricci curvature of a compact Kähler manifold and the complex Monge-Ampère equation. I, Communications on Pure and Applied Mathematics 31 (3): 339-411 (1978), doi:10.1002/cpa.3160310304, MR 480350

[Yeung] S.-K. Yeung, Geometry of domains with the uniform squeezing property, Advances in Mathematics 221 (2009), 547-569. 